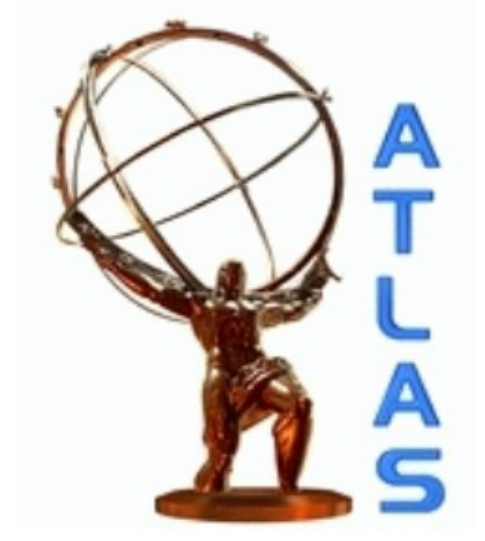

\title{
$R$-Parity violation with jet signatures at the ATLAS Detector
}

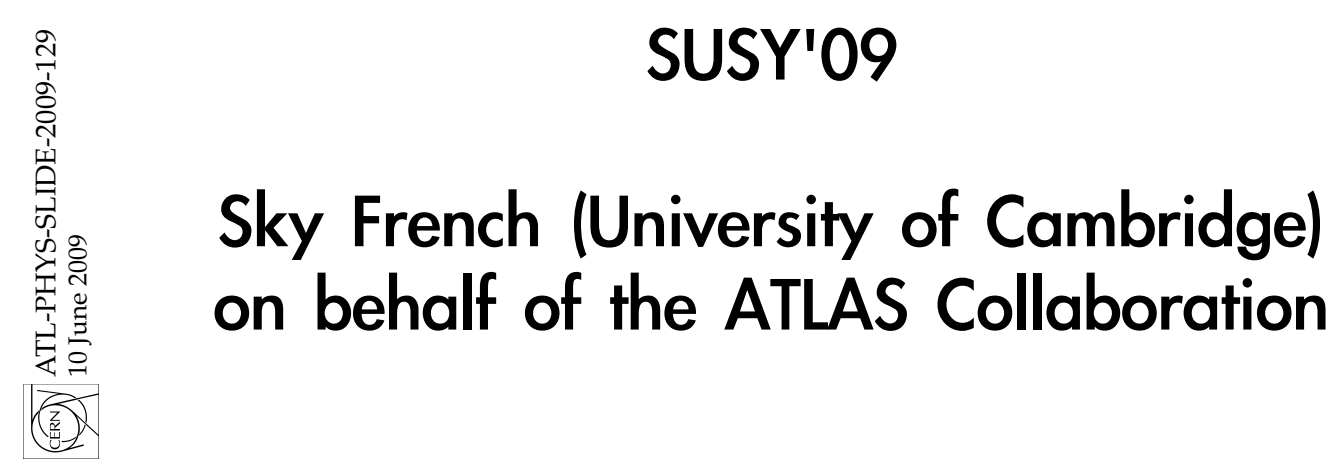

Sky French 


\section{Introduction (1)}

- J.M. Butterworth et al. have recently proposed a method for discovering heavy particles ...

by considering jet substructure - looking at very boosted particles and trying to spot the single jets which are made out of collimated subjets...

[see J. Butterworth, J. Ellis, A. Raklev and G. Salam, Discovering baryon-number violating neutralino decays at the LHC, arXiv:0906.0728 and also J. Butterworth, J. Ellis and A. Raklev, hep-ph/0702150]

- This method looks for massive particles with a boost decaying to three semi-collimated light jets

- They use the $\mathbf{k}_{\mathrm{T}}$ algorithm to look at jet substructure and demonstrate the method by searching for neutralino decays in baryon-number violating SUSY

- We test their approach for use at ATLAS by also considering hadronic neutralino decays... 


\section{Introduction (2)}

- We take the explicit R-Parity violating terms of the supersymmetric Lagrangian to be the following allowed in soft SUSY breaking:

$$
W=\frac{1}{2} \lambda^{i j k} L_{i} L_{j} \bar{e}_{k}+\lambda^{\prime i j k} L_{i} Q_{j} \bar{d}_{k}+\mu^{\prime i} L_{i} H_{u}+\frac{1}{2} \lambda^{\prime \prime i j k} \bar{u}_{i} \bar{d}_{j} \bar{d}_{k}
$$

- Non zero values of any of these couplings give us very different phenomenologies to the RPC MSSM

- We consider non-zero $\lambda$ " which is the dominant parameter in baryon number violating models

- It will give us the decay:

$$
\tilde{\chi}_{1}^{0} \rightarrow q q q
$$

- In this new method we do not need to use a lepton requirement to separate these decays from a highly hadronic background as has previously been the case...

[see: B.C. Allanach et al, Measuring Supersymmetric Particle Masses at the LHC in Scenarios with Baryon-Number R-Parity Violating Couplings, hep-ph/0102173] 


\section{Method (1)}

- In this method the primary tool for classifying jet substructure is the $k_{T}$ algorithm

- Takes a pair of calorimeter clusters $(k, l)$ and calculates two resolution variables:

$$
\begin{gathered}
d_{k B}=p_{T k}^{2} \\
d_{k l}=\min \left(p_{T k}^{2}, p_{T l}^{2}\right) R_{k l}^{2} / R^{2}
\end{gathered}
$$

- If it finds that $d_{k l}$ is smallest $k$ and I will be combined to make a single object with momentum $p_{k l}$

$$
\begin{gathered}
R_{k l}^{2}=\left(\eta_{k}-\eta_{l}\right)^{2}+\left(\phi_{k}-\phi_{l}\right)^{2} \\
p_{k l}=p_{k}+p_{l}
\end{gathered}
$$

- The scale at which jets from two or more collimated partons separate into their subjets is reflected by the values of $d_{k l}$ calculated in this jet-finding algorithm

- The variable $y$ is then introduced:

$$
y=d_{k l} / m^{2}
$$

Sky French

on behalf of the ATLAS Collaboration 


\section{Method (2)}

- If the heavy particles, in our case neutralinos, are sufficiently boosted the three jets from the decay will collimate...

- Look at the true circumcircle radius (or half the maximum $\Delta R$ when this is smallest) of the three jets as a function of particle $p_{T}$

- This gives some indication of an appropriate $\mathbf{R}$ parameter

higher $\mathbf{R}$ worsens jet reconstruction but improves signal efficiency

- Jet mass defined as the invariant mass of the final objects

Sky French on behalf of the ATLAS Collaboration

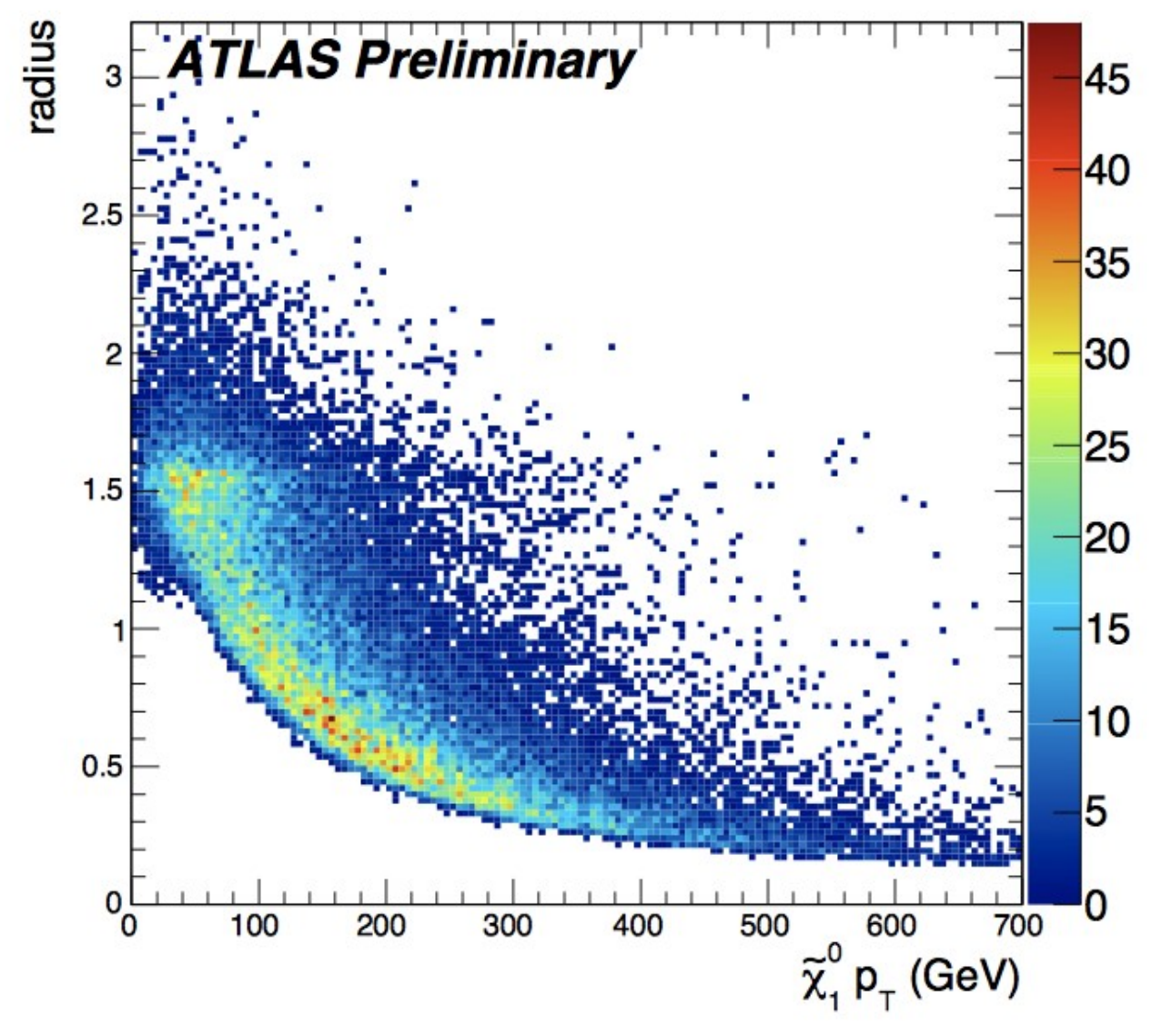


- Choose R parameter of 0.7

\section{Method (3)}

- $y_{1}$ and $y_{2}$ are the $y$-values from the last and next to last merging of the subjets

- Triggers:

- signal events have multiple high $p_{T}$ jets

- high trigger efficiency for many un-prescaled triggers e.g. 4 jets with $p_{T}>125 \mathrm{GeV}$ trigger

- Make the following offline selection cuts:

1. at least four jets in the event, with the four highest $p_{T}$ jets having $|\eta|<2.5$

2. two jets with $p_{T}>275 \mathrm{GeV}$

3. for both of these jets $y_{2}>-0.17 y_{1}+0.08$

4. two further jets both with $p_{T}>135 \mathrm{GeV}$

\begin{tabular}{|c|c|c|c|c|}
\hline & Cut 1 & Cuts $1 \& 2$ & Cuts $1,2 \& 3$ & Cuts $1,2,3 \& 4$ \\
\hline SUSY & 14577 & 5849 & 792 & 347 \\
\hline$t \bar{t}$ & $2.3 \times 10^{5}$ & 2,000 & 200 & 1 \\
\hline dijets & $3.2 \times 10^{9}$ & $1.1 \times 10^{6}$ & $1.8 \times 10^{4}$ & 1100 \\
\hline$W+$ jets & 1500 & 20 & 20 & 0 \\
\hline$Z+$ jets & $1.9 \times 10^{4}$ & 210 & 2 & 1 \\
\hline
\end{tabular}




\section{The Model}

- Take the SPS1a SUSY Point as our straw model to test the method and set $\lambda "{ }_{112}=0.001$ giving a final decay with no heavy flavours or displaced vertices to aid identification

- Consider $1 \mathrm{fb}^{-1}$ of integrated luminosity at $10 \mathrm{TeV}$

- Signal Model: $\lambda{ }^{\prime 12}=0.001, \sigma=17 \mathrm{pb}$ (at LO), $\mathrm{m}_{\text {neutralino }}=96.1 \mathrm{GeV}, \mathrm{m}_{0}=100 \mathrm{GeV}$,

$$
\tan \beta=10, A_{0}=-100, m_{1 / 2}=250 \mathrm{GeV}, \operatorname{sign}(\mu)>0
$$

(NB: ruled out by current experimental limits, but this analysis should be model independent and apply to other $\lambda^{\prime \prime}{ }_{i j k}$ with $\left.\mathrm{i}, \mathrm{j}, \mathrm{k}=1,2\right)$

- Backgrounds: W+jets, Z+jets, dijets and ttbar

- Signal events were generated using Pythia

- Both signal and background were then passed through full simulation of the ATLAS detector

- The $\mathrm{k}_{\mathrm{T}}$ algorithm was used on locally calibrated topological clusters built from calorimeter cells 


\section{The ATLAS Detector}

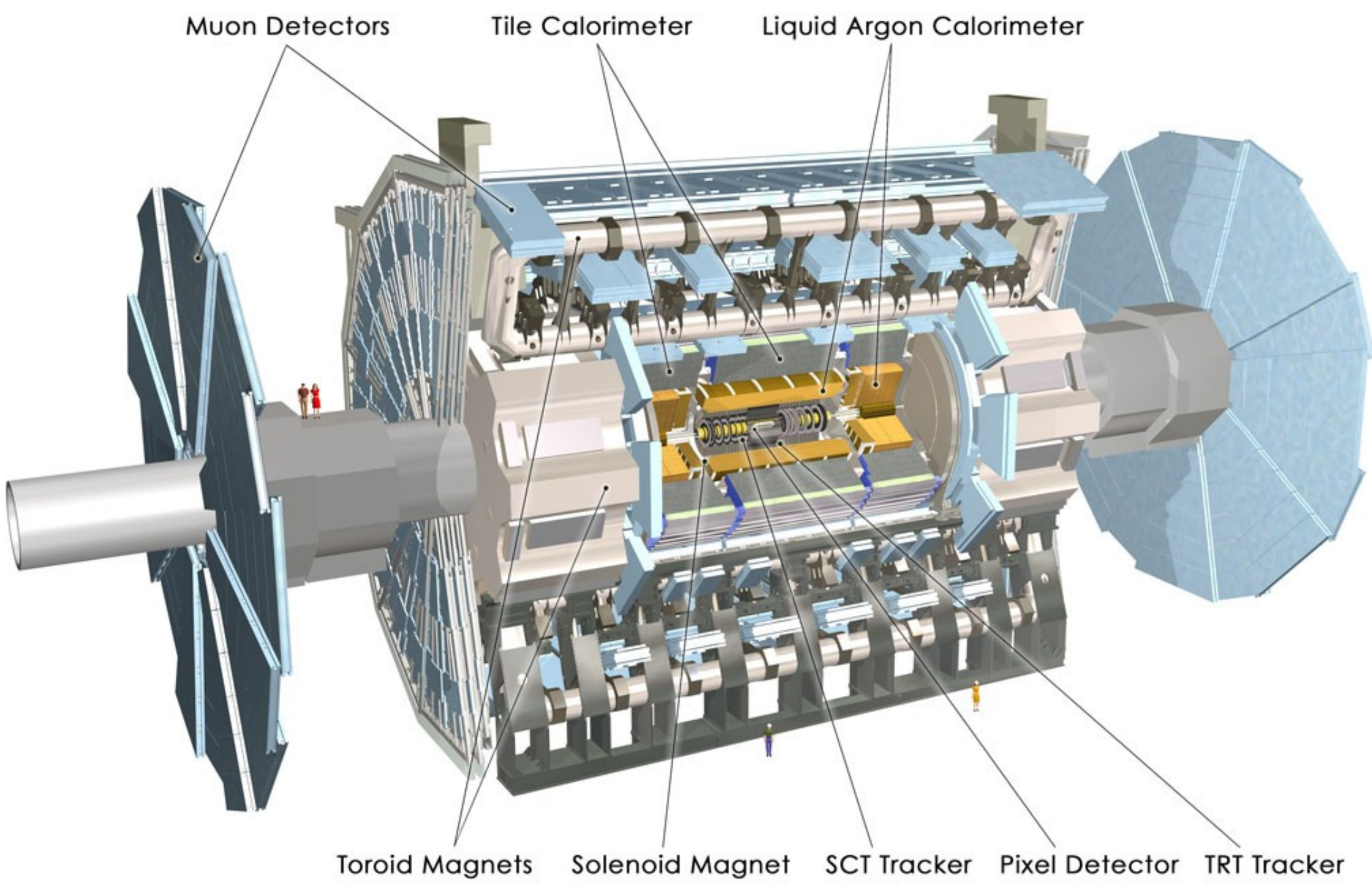

Sky French

on behalf of the ATLAS Collaboration 


\section{Results (1)}

- The signal $\mathrm{p}_{\mathrm{T}}$ vs. mass distribution shows a stripe at a mass of $\sim 100 \mathrm{GeV}$ (background distribution shows no such structure) which validates the strategy of selecting neutralino jets using a high $\mathrm{p}_{\mathrm{T}}$ cut
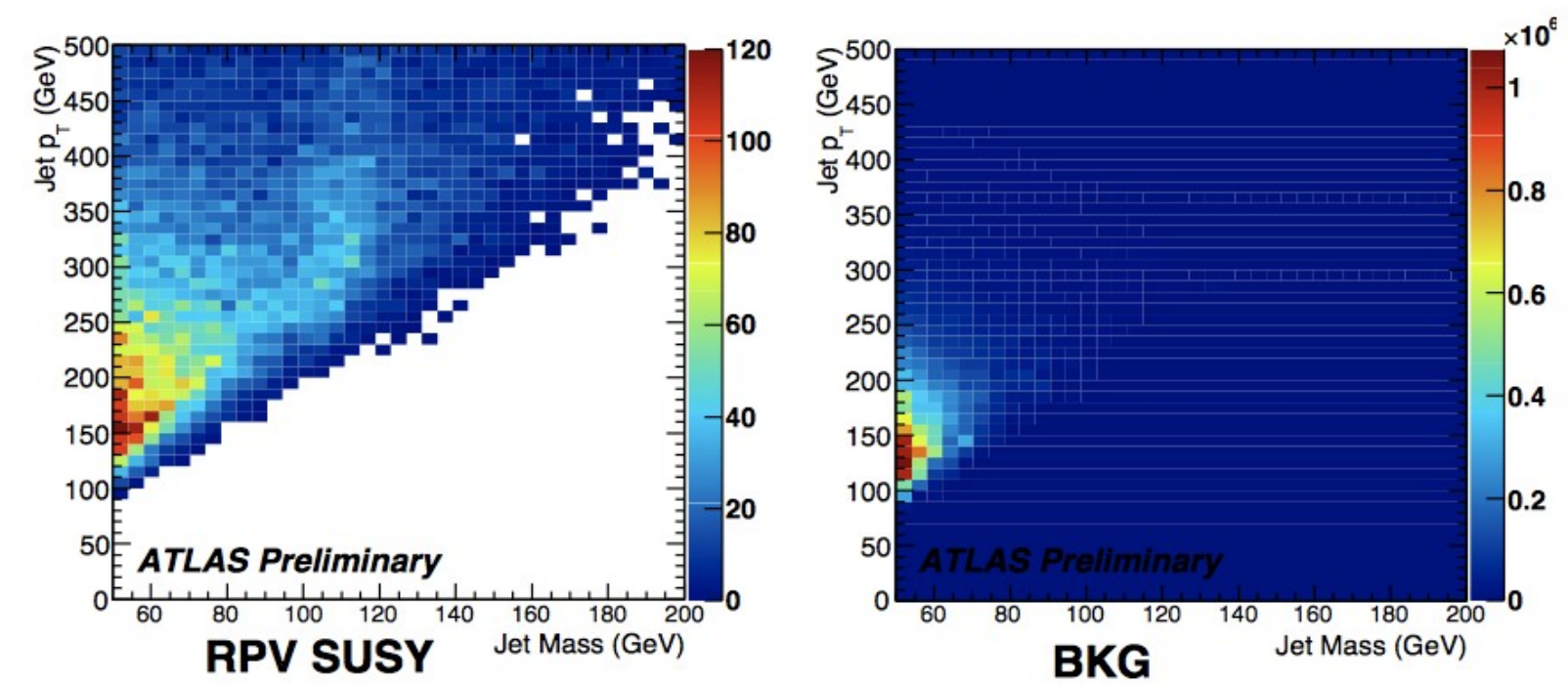

- The mass distribution of jets with $p_{\mathrm{T}}>\mathbf{2 7 5} \mathrm{GeV}$ shows that we must reduce $Q C D$ background by several orders of magnitude in order to see the signal neutralino peak

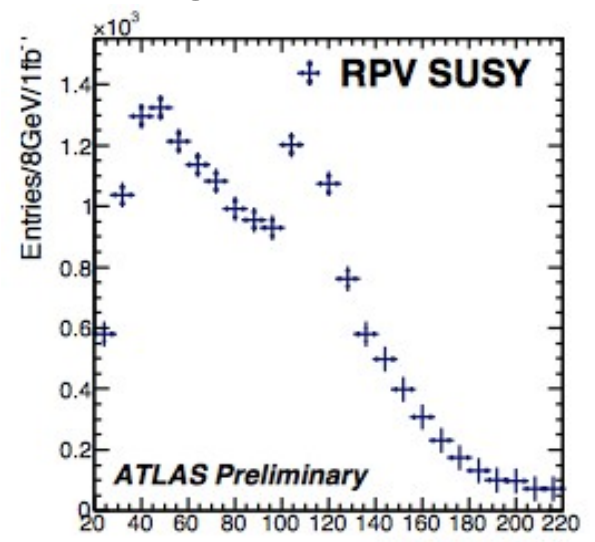

Sky French

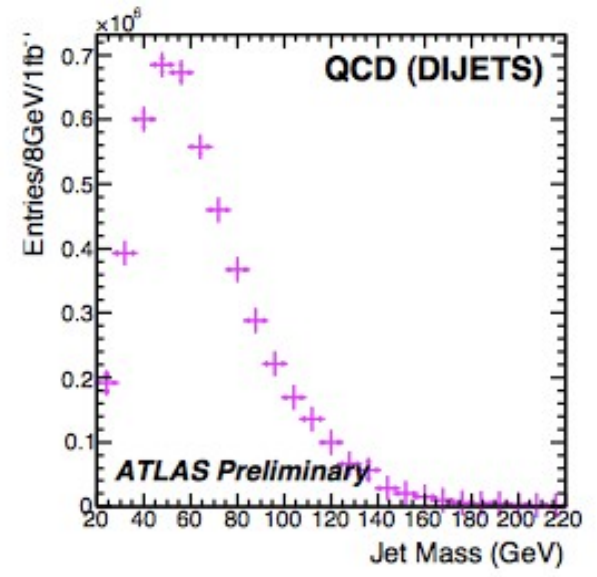

Jet Mass $(\mathrm{GeV})$

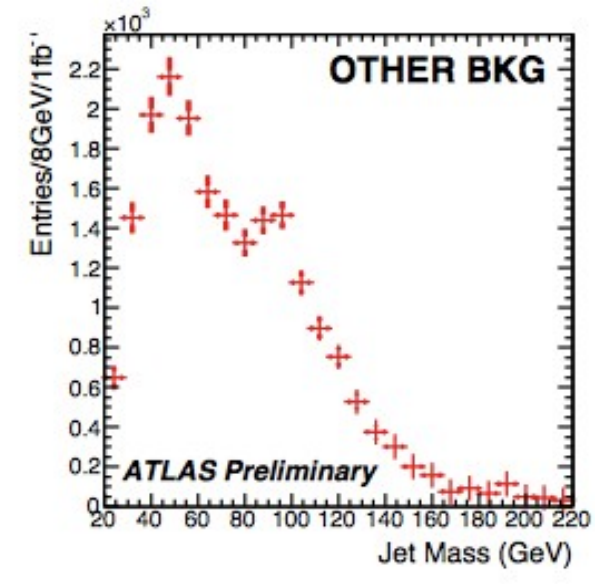




\section{Results (2)}

- A plot of $y_{2}$ vs $y_{1}$ shows that a cut on these two variables will reduce the dijet background significantly (events above the drawn cut line are accepted)

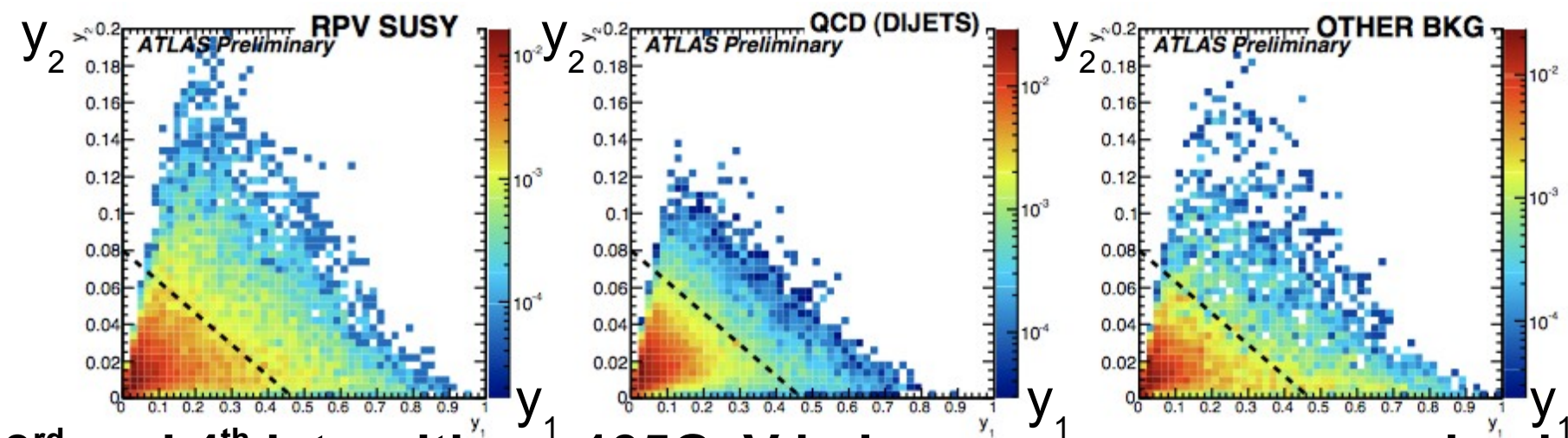

- Requiring $3^{\text {rd }}$ and $4^{\text {th }}$ jets with $p_{T}>135 \mathrm{GeV}$ helps remove even more background without reducing signal ( $40 \%$ of neutralinos produced by squark decay - expect another jet, or even a jet from higher up a decay chain)

- The mass distribution of jets with $p_{T}>275 \mathrm{GeV}$ and $y_{2}>-0.17 y_{1}+0.08$ after making all event selection cuts shows that QCD has been reduced by $10^{3}$ across the jet mass range
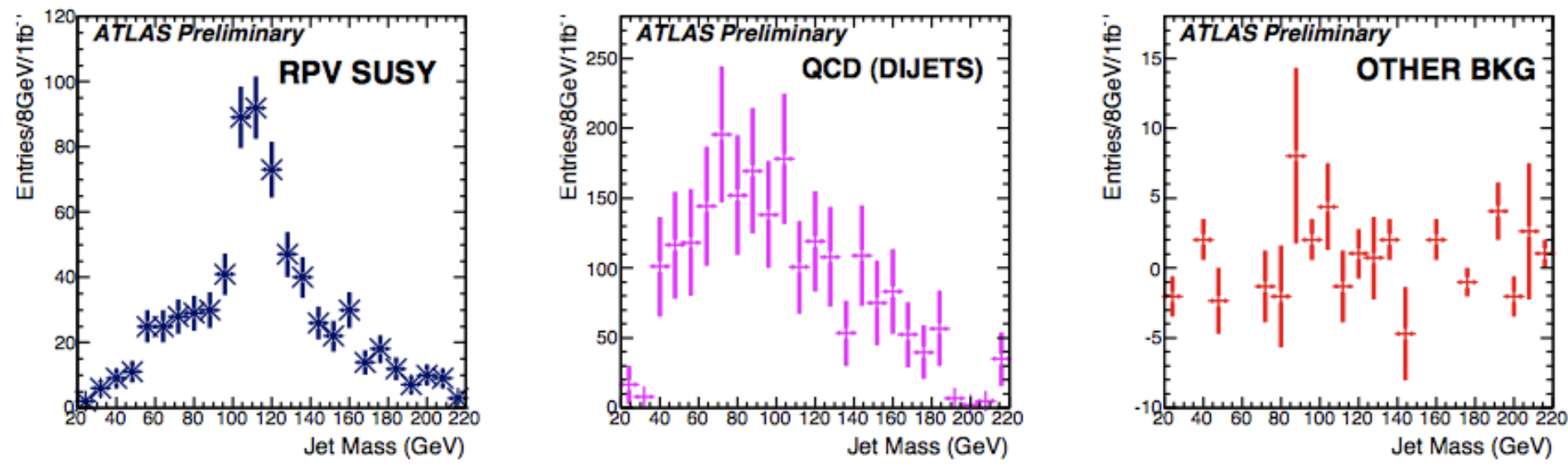

Sky French 


\section{Results (3)}

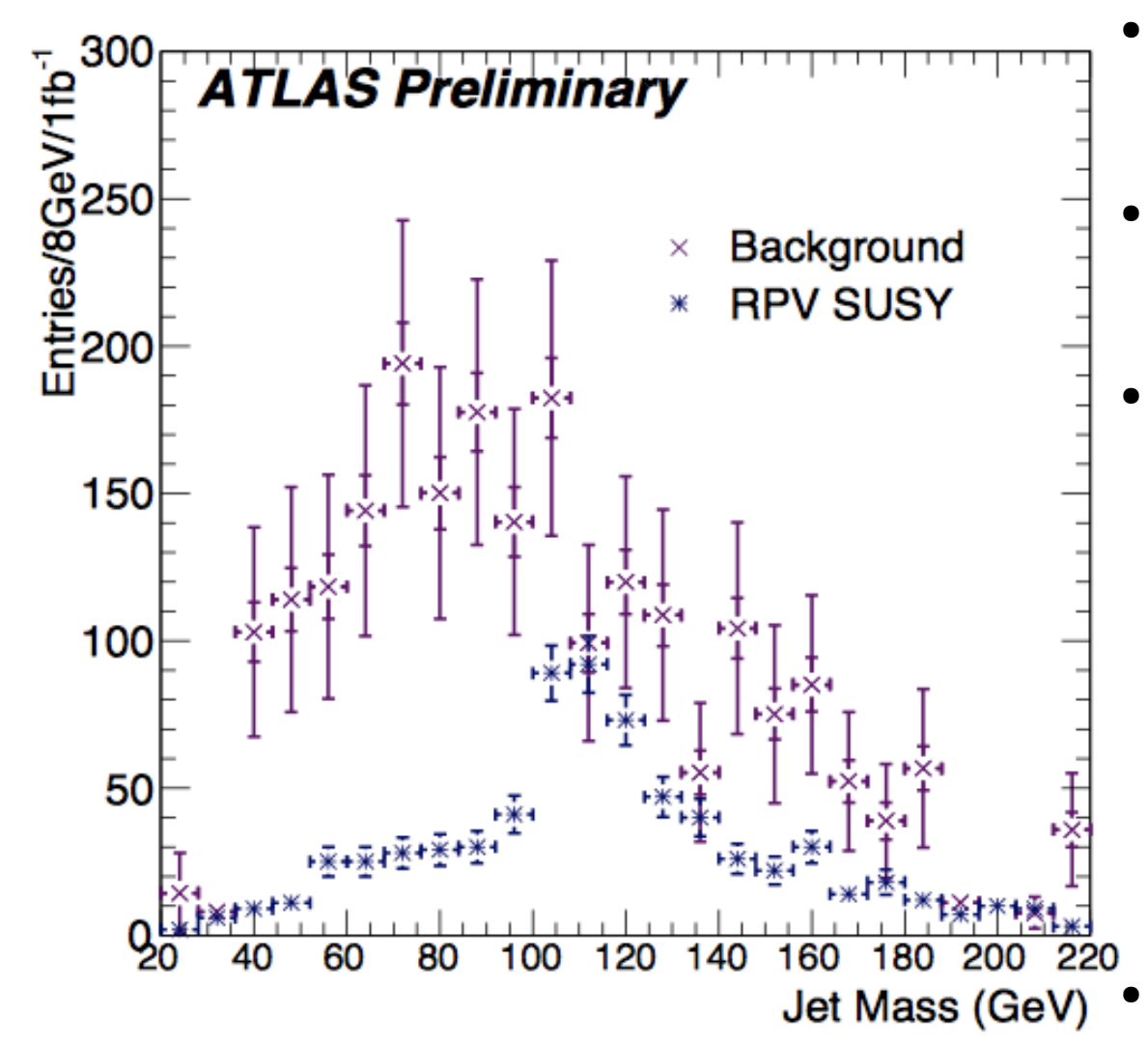

Sky French on behalf of the ATLAS Collaboration
- Comparable numbers of signal and background events remaining

- Remains a clear peak in the signal distribution at the mass of the neutralino

- Cut on y-values has significantly reduced the dijet background

- QCD mass distribution suffers from low statistics, due to limited Monte Carlo, and so the events are highly weighted

- In real data this will not be a problem

- The smaller error bars (poisson) give the size of the errors expected for $1 \mathrm{fb}^{-1}$ of data, these are much more manageable

Success or failure of this method depends on the amount of background underneath the signal and on the degree to which we can estimate or measure its size and shape

- We might be able to model this background by loosening cuts and letting in more background to make background enriched samples - allowing us to ascertain the true shape and smoothness (or otherwise) of the underlying background 


\section{Conclusions \& Outlook}

- We have successfully taken a recently proposed method and demonstrated that, albeit with a few additional background removing cuts, ATLAS has the potential to detect highly boosted neutralinos by looking at the substructure of the resulting single collimated jets

- We could extend this to consider other jet algorithms

- We have simulated and studied an R-Parity violating supserymmetric point but we have avoided making the analysis depend strongly on this choice... but it is 'aided' by:

- Supersymmetric pair production providing a natural source of neutralino pairs (allowing us to ask for two high $\mathrm{p}_{\mathrm{T}}$ candidates)

- Long decay chains providing source of boosts and additional jets ( $3^{\text {rd }}$ and $4^{\text {th }}$ )

We have therefore only tested the applicability of this method to models with pair production of the decaying state and models where we have an equivalent means of providing the part of the background rejection provided by the $3^{\text {rd }}$ and $4^{\text {th }}$ jet cuts

- These cuts have not been fully optimised but serve to demonstrate the suitability of the method for investigating baryon number violating SUSY models in ATLAS 